\title{
Curva de rendimiento y composición de leche en ovejas criollas de la Montaña de Guerrero, México
}

\section{Yield and milk composition curve in the creole ewe from la Montaña de Guerrero, Mexico}

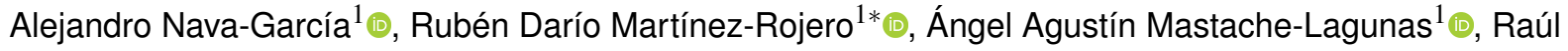 \\ Ulloa-Arvizu² ${ }^{2}$ \\ ${ }^{1}$ Colegio Superior Agropecuario del Estado de Guerrero. Av. Vicente Guerrero 81, Centro, CP. 40000. Iguala, Guerrero, México. \\ ${ }^{2}$ Facultad de Medicina Veterinaria y Zootecnia, Universidad Nacional Autónoma de México. Av. Universidad 3000, UNAM-CU, CP. 04510. \\ Ciudad de México, México. \\ *Autor de correspondencia: rubendariomr1@prodigy.net.mx
}

Nota científica recibida: 06 de septiembre de 2018 aceptada: 16 de febrero de 2019

RESUMEN. El objetivo fue estimar la curva de producción de leche y determinar sus componentes químicos en ovejas Criollas Obispo. Se procesaron datos de producción de 22 ovejas con el modelo de Wood; los registros se realizaron cada semana con el método de amamantamiento-pesaje de la cría-ordeño y la determinación de la composición química se hizo con un equipo Lactichek. El pico máximo promedio de producción (539.60 g) se alcanzó en la segunda semana, con un rendimiento diario de $342.82 \pm 11.59 \mathrm{~g}$ y producción acumulada por oveja estimada para una lactancia de 12 semanas de $30.58 \pm 1.21 \mathrm{~kg}$. Los promedios para proteína, grasa, lactosa, sólidos totales y sólidos no grasos fueron de $5.71 \%$, $2.49 \%, 4.59 \%, 13.66 \%$ y $11.24 \%$, respectivamente. La curva de producción de leche en ovejas Obispo registra su máximo incremento entre la segunda y la tercera semana posparto.

Palabras clave: Borrega, lactancia, Obispo, posparto.

ABSTRACT. The objective was estimate the yield curve and to determine the composition of the milk in Creole Obispo ewes. Milk production data of 22 ewes were processed utilizing the Wood model; dates were registered weekly using the lamb suckling-lamb weighing-milk method, while for their milk composition determination an equipment "Lactichek" was utilized. the higher mean peak of milk production $(539.60 \mathrm{~g})$ was registered into the second week, while the daily performance was of $342.82 \pm 11.59 \mathrm{~g}$ and the accumulated milk production estimation per ewe for a lactation of 12 weeks was of 30.58 $\pm 1.21 \mathrm{~kg}$. The average values of protein, fat, lactose, total solids, no fat solids, water and density were $5.71 \%, 2.49 \%$, $4.59 \%, 13.66 \%$ and $11.24 \%$, respectively. The milk production curve in the "Obispo" ewe register its highest rising between the second and the third weeks post-lambing.

Key words: Lactation, Obispo, post-lambing, sheep.

\section{INTRODUCCIÓN}

Debido a que en el México prehispánico no existían animales domésticos, los conquistadores trajeron las primeras razas de ovinos de tipo lanar (Merino, Manchega, Churra y Lacha) que durante siglos desarrollaron adaptación a condiciones difíciles de subsistencia a través de mecanismos de selección natural y dieron originen a animales Criollos, con valores productivos diferentes a los de sus predecesores (Solís et al. 2015). En la Región de la Montaña de Guerrero se encuentra un ovino Criollo lanado de vellón blanco o negro conocido como borrego Obispo, Diablo o de Cuatro Cuernos (mutación denominada policerismo) que, por ser un animal rústico con adaptación a condiciones precarias de cría, constituye un recurso genético que ha pasado inadvertido por siglos y en peligro de desaparecer, debido a que no se han realizados esfuerzos para estudiarlo y conservarlo (Martínez 2016). En este sentido, se desconocen aspectos básicos de su comportamiento reproductivo, producción y composición química de 
la leche. Los mamíferos presentan curvas en la producción de leche, que satisfacen las necesidades nutricionales de sus crías, cuyo crecimiento durante la primera etapa de su vida depende de la producción láctea de la madre (Bencini y Pulina 1997, Delgadillo 2016). En el ovino, representa el $75 \%$ del peso al destete del cordero y se ha determinado correlación significativa entre la secreción de leche de la madre y la ganancia de peso de la cría, por lo que la capacidad lechera de una oveja es un factor fundamental para destetar corderos más pesados (Benchohra et al. 2013, Ochepo et al. 2015). En el caso particular de los rumiantes esta dependencia es casi absoluta durante el primer mes de vida y a partir de entonces comienza a disminuir, debido a la menor producción láctea de la madre y al inicio del consumo de alimentos sólidos por el cordero (Castellanos y Valencia 1982). En los sistemas de producción, las curvas de lactancia se utilizan para llevar a cabo programas de mejoramiento genético, o bien para aplicaciones técnicas, como determinar la edad al destete de las crías. Adicionalmente, el conocimiento de la composición de la leche es de interés para determinar su calidad nutritiva y otras propiedades. Considerando lo anterior, el objetivo fue estimar la curva de producción durante los tres primeros meses de lactancia y determinar la producción y la composición química de leche en un rebaño de ovejas Criollas de la Montaña de Guerrero.

\section{MATERIALES Y MÉTODOS}

El estudio tuvo una duración de 12 meses, se realizó en el Colegio Superior Agropecuario del Estado de Guerrero (CSAEGRO), situado en el km 14.5 de la carretera Iguala-Cocula, Guerrero, a $18^{\circ} 15^{\prime} 52^{\prime \prime}$ LN y $99^{\circ} 38^{\prime} 52^{\prime \prime}$ LO, a una altura de 640 metros sobre el nivel del mar. El clima es tipo AW0 (w) (i') g (el más seco de los climas semi-húmedos), con precipitación pluvial durante los meses de junio a octubre de 797 $\mathrm{mm}$ y temperatura promedio anual de $25.7^{\circ} \mathrm{C}$ (García 1988). Se utilizaron 22 ovejas Criollas multíparas en su segundo parto de dos a tres años de edad con peso promedio de $29.7 \mathrm{~kg}$, que se manejaron en un sistema de semi-estabulación (Figura 1); du- rante las mañanas (07:00-15:00 h) salían a pastorear en praderas con pasto insurgente (Brachiaria brizantha $\mathrm{H}$.), pasto Mombasa (Panicum máximum) y pasto nativo compuesto principalmente de zacate remolino (Paspalum notatum). Por la tarde-noche se estabularon en corrales techados en donde recibieron por oveja $200 \mathrm{~g}$ de concentrado comercial con $12 \%$ de proteína cruda (PC) y $3500 \mathrm{kcal} \mathrm{kg}^{-1}$, más agua ad libitum.

Se utilizó un empadre estacional con duración de cuatro semanas, implementado durante el otoño de 2016. Las ovejas se aparearon con machos Obispo mediante monta natural dirigida, previa detección de estros con un macho recelador vasectomizado, se anotó la fecha del servicio y cinco meses después, en la primavera de 2017, se registraron los partos. Todos fueron sencillos y se concentraron dentro de un periodo de tres semanas. Una vez que la cría mamó calostro, la producción de leche se midió cada semana a partir al tercer día después del parto, hasta que los corderos se destetaron a los tres meses de edad. Para estimar la producción de leche se utilizó el método propuesto por Doney et al. (1979) de peso de la cría-amamantamiento-peso de la cría, más la ordeña manual de la leche residual. Con este propósito un día antes del muestreo (15:00 h), los corderos se separaron de sus madres dentro de una corraleta adyacente para que estuvieran contacto directo con ellas, pero impidiéndoles que mamaran por el resto de la tarde y noche, para estimar la producción de leche por día de la borrega a la mañana siguiente. Un primer pesaje de las crías (Peso 1) se registró a las 07:00 $\mathrm{h}$ antes de reunirlas con sus madres para que lactaran durante aproximadamente 30 minutos, y pesarlas de forma inmediata (Peso 2) antes de separarlas por segunda vez de su madre por el resto de la mañana y parte de la tarde (07:30 a 15:00 h). La diferencia entre los pesos 1 y 2 registrados se consideró como la cantidad de leche producida consumida por el cordero (Muestreo 1). Inmediatamente después de amamantar a sus crías las ovejas se ordeñaron y se pesó la leche residual en una báscula digital para sumarse al valor obtenido de la diferencia de peso del cordero antes y después de amamantarlo. Este procedimiento 


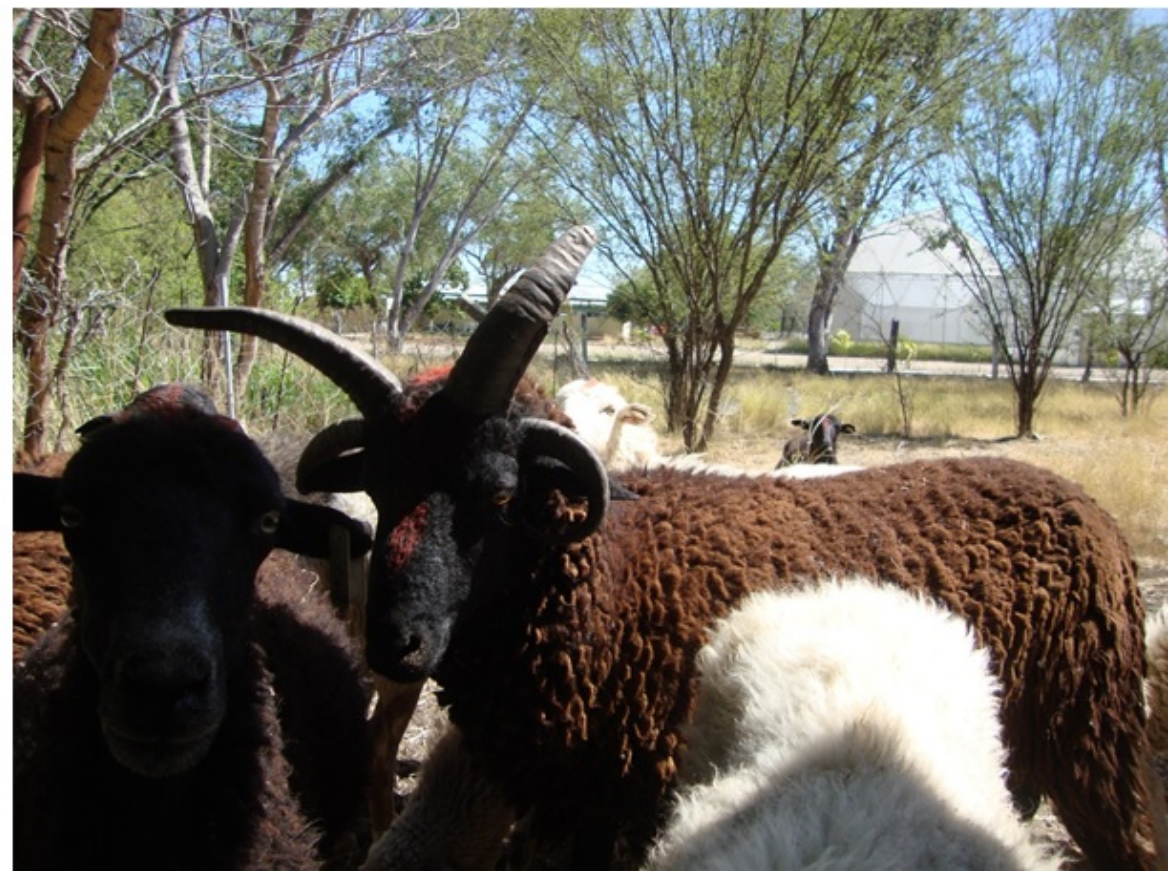

Figura 1. El borrego Criollo Obispo, Diablo o Cuatro Cuernos de la Montaña de Guerrero.

del pesaje-amamantamiento-ordeño se repitió por la tarde $(15: 00 \mathrm{~h})$, después de que las madres regresaron del pastoreo y se concentraron en los corrales para recibir alimento concentrado (Muestreo 2). La suma del Muestreo 1 y Muestreo 2 (pesaje de la mañana más pesaje de la tarde), más la ordeña residual se consideró como la leche producida por la oveja en $24 \mathrm{~h}$.

Para determinar la composición química de la leche, los valores de grasa (\%), sólidos no grasos (\%), sólidos totales (\%), proteína (\%) y lactosa (\%) se obtuvieron por análisis automatizado basado en tecnología espectométrica de ultrasonido utilizando muestras de leche de $40 \mathrm{~mL}$ por oveja tomada por la mañana en un equipo Lacti Check digitalizado (Modelo LC-01/R cow-sheep, Page \& Pedersen International Ltd) calibrado en modo sheep en porcentaje.

Para la composición de la leche se utilizó estadística descriptiva (Steel y Torrie 1989) y para caracterizar la curva de lactancia y estimar su producción se utilizó la función gama incompleta $Y_{t}$ $=a t^{b} \mathrm{e}^{-c t}$ (Wood 1976), modelo que ha sido utilizado en ovinos por diferentes investigadores obteniendo valores altos de persistencia y de $\mathrm{R}^{2}$ (Chang et al. 2001, Chang et al. 2002, Keskin y Dağ 2006). Donde $Y_{t}$ es la producción en el t-ésimo día de la lactancia (días después del parto); e = base del logaritmo natural; $a, b$ y $c=$ son constantes donde a representa un factor de escala, o producción de leche al inicio de la lactancia, mientras que b y c constituyen la declinación límite de la curva antes y después del pico de la lactancia.

La producción total de leche se estimó sumando los valores diarios generados con el modelo de Wood que fue construido utilizando los registros semanales de producción. La estimación de los parámetros del modelo se obtuvo por el método de Levenberg-Marquardt implementado en el procedimiento NLIN del paquete estadístico (SAS 2016). Se usó esta metodología debido a que tiene la capacidad de buscar, dependiendo la distancia donde se encuentre la solución actual del mínimo local; si está lejos, se comporta como el método de máximo descenso aunque es un proceso lento logra la convergencia; y cuando la solución está cercana al mínimo local, el método presenta un comportamiento pare- 
cido a Gauss-Newton exhibiendo una rápida convergencia pero evitando los problemas cuando la matriz jacobina es no singular (Cornejo y Rebolledo 2016).

\section{RESULTADOS Y DISCUSIÓN}

\section{Producción de leche y pico de lactancia}

Las ovejas Obispo no engendraron partos gemelares. En la Figura 2 se aprecia la producción ajustada de leche durante los primeros tres meses posparto con el modelo de Wood (1976). El valor máximo promedio de producción fue de $539.3 \mathrm{~g}$ que se alcanzó el día 8 (b/c) de la lactancia, para posteriormente disminuir a $160.3 \mathrm{~g}$ en el día 90 posparto. Esto implica que la persistencia, determinada por las diferencias entre la producción de leche a partir del día 8 de la lactación y el día 90 posparto, fue de 379 g, es decir una reducción de 4.62 g por día. La producción acumulada de leche estimada en una lactancia de 12 semanas fue de $30.58 \pm 1.21 \mathrm{~kg}$, con producción diaria de $342.82 \pm 11.59 \mathrm{~g}$ (Tabla 1).

La mayoría de los estudios relacionados con la producción de leche en ovejas, se han realizado en razas lecheras y son pocos los trabajos en razas con aptitud zootécnica para carne y/o lana. En este sentido, la adaptación de las ovejas de razas lecheras al ordeño manual o mecánico ha permitido establecer valores fiables de la cantidad de leche producida, ya que puede pesarse todos los días una vez extraída. Por el contrario, en las razas de carne o de lana se ha tenido que recurrir a diversos procedimientos para estimar la producción de leche, ya que las ovejas normalmente amamantan a sus corderos. Entre estos métodos se incluye el empleado en el presente estudio, que consiste en registrar la diferencia de peso de los corderos antes y después de mamar para estimar la cantidad de leche consumida por día en un determinado número de intervalos de tiempo (Doney et al. 1979).

Los trabajos realizados por Bencini y Purvis (1990), Reynolds y Brown (1991) y Bencini et al. (1992) han observado que la producción lechera en la oveja aumenta en las primeras semanas posparto, alcanzando el pico máximo en la segunda y tercera semana de lactación, después de las cuales la curva de producción declina hasta el secado de la hembra dependiendo de la raza, el genotipo y el potencial lechero individual. Los resultados obtenidos presentan similitud con los pocos trabajos que se han realizado en México en ovejas Criollas quienes reportan una producción de leche diaria de $200 \mathrm{~mL}$ en un ordeño con una producción total en 110 días estimada de $22 \mathrm{~L}$, con pico de lactancia de 11 días posparto y persistencia de 110 días (Pedraza et al. 1992). Peralta-Lailson et al. (2005) reportan una producción diaria de leche por oveja de $174 \mathrm{~mL}$ en un solo ordeño (matutino), con pico de lactación en la variedad negra el día cuatro después del parto, mientras que en las variedades blanca y café no se observa pico, ya que la curva fue descendiendo con producción total por hembra de entre 11 y $13 \mathrm{~L}$ en 120 días de ordeño. Al respecto Pedraza et al. (1992) señalan que los borregos Chiapas son animales ligeros poco productivos, si se comparan con sus antecesoras españoles, los cuales llegan a pesar hasta $70 \mathrm{~kg}$, producir $3 \mathrm{~kg}$ de lana y más de un litro de leche diario. Por lo que se puede inferir que las diferencias en el peso y la productividad de los animales son el resultado de un largo proceso de adaptación a las condiciones ambientales y geográficas. Estos investigadores argumentan también que la talla y la productividad de las razas españolas podrían ser el producto de un proceso continuo de selección, dirigido a que dichas razas se especializaran. De este modo, considerando el prolongado aislamiento de la región de los Altos de Chiapas, cabría entonces pensar también, de acuerdo con Pedraza et al. (1992), que el borrego Chiapas, de talla pequeña y de escasa producción de leche y de lana burda y gruesa, es más parecido a las ovejas introducidas en la Nueva España a principios del siglo $\mathrm{XVI}$, que a las propias razas españolas de hoy en día. Siguiendo este razonamiento, es posible que una situación similar pudiera haber ocurrido en el caso de la oveja Criolla Obispo que se ha mantenido aislada por siglos en la región de la Montaña de Guerrero.

Aunque con una menor producción, la oveja Obispo presenta una curva de lactancia parecida a la obtenida en ovinos de doble propósito de otras regiones del país sin aptitud zootécnica lechera. En el 


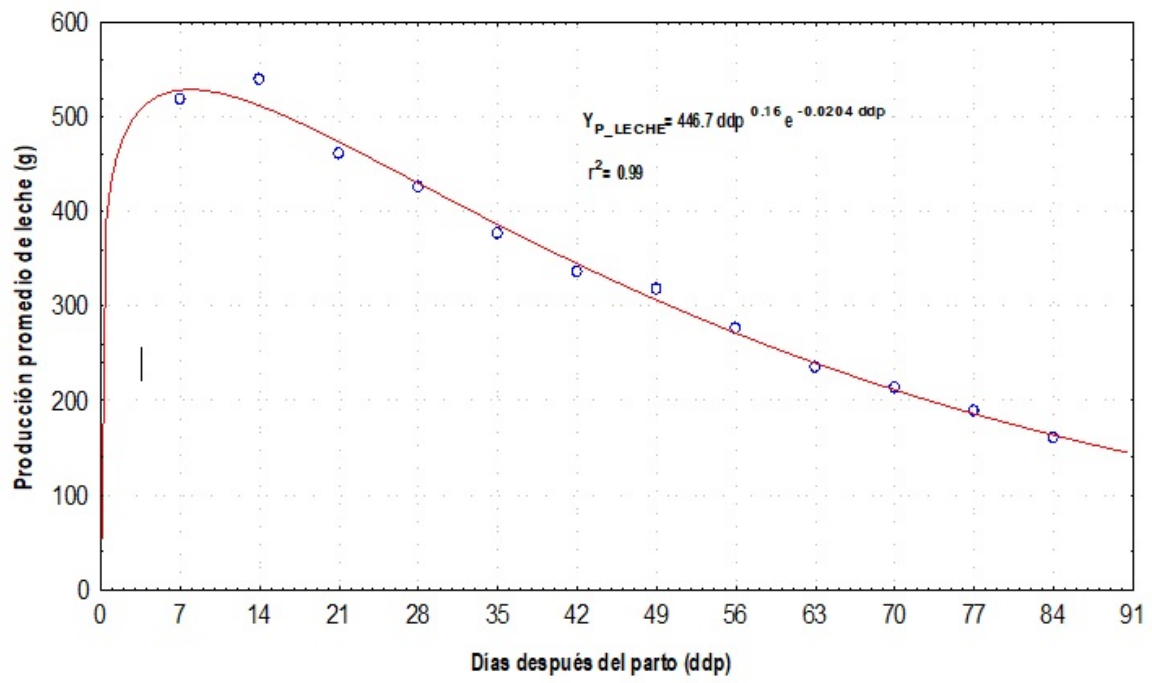

Figura 2. Producción de leche registrada cada semana en ovejas Criollas Obispo durante los primeros tres meses de lactancia, ajustada mediante el Modelo de Wood (1976).

Tabla 1. Promedio ( \pm error estándar) y parámetros del modelo gama incompleto para la producción de la leche de ovejas Criollas Obispo en lactancia de 12 semanas.

\begin{tabular}{lcccccc}
\hline Característica & $\mathrm{a}$ & $\mathrm{b}$ & $\mathrm{c}$ & $\mathrm{r}^{2}$ & Producción diaria $(\mathrm{g})$ & Producción acumulada $(\mathrm{kg})$ \\
\hline Leche & 446.7 & 0.16 & -0.0204 & 0.99 & $342.82 \pm 11.59$ & $30.36 \pm 1.21$ \\
\hline $\mathrm{a}=$ factor de escala, o producción de leche al inicio de la lactancia, $\mathrm{b}=$ declinación límite de la curva antes de \\
la lactancia, y $\mathrm{c}=$ declinación límite después del pico de la lactancia.
\end{tabular}

altiplano de México Ochoa et al. (2002) calcularon que la máxima producción de leche en ovejas Rambouillet se obtuvo en la semana tres, cuatro y cinco después del parto, con rendimientos cercanos a los $1000 \mathrm{~mL}$, mientras que el pico de producción ocurrió en el día 19 de lactación con promedio de $998 \mathrm{~mL}$ y producción por lactancia de $71.0 \mathrm{~kg}$. Valores que son mayores si se compara con el rendimiento registrado en este estudio para la oveja Obispo. Al respecto se sabe que la dimensión, forma de la ubre y del pezón están relacionadas con la producción de leche en la oveja (Nudda et al. 2000, Fasulkov 2012); entre más grande es la glándula, la cantidad de parénquima con tejido secretor de leche es mayor, por lo que el volumen de producción guarda una relación directa con el tamaño de la ubre (Labussière 1988). En el caso particular de la oveja Obispo, es evidente la relación entre la baja producción de leche y las características anatómicas de su glándula mamaria, ya que este genotipo presenta una ubre pequeña y recogida con pezones divergentes y cortos que es propicia para evitar lesiones durante su pastoreo en áreas con matorrales espinosos (Martínez et al. 2016).

\section{Composición de la leche}

En la Tabla 2 se muestran los valores promedio de la composición química de la leche de ovejas Criollas Obispo. El contenido de proteína cruda, grasa, lactosa, sólidos totales y sólidos no grasos fueron de $5.73 \pm 0.07 \%, 2.49 \pm 0.19 \%, 4.59 \pm$ $0.01 \%, 13.66 \pm 0.19 \%$ y $11.24 \pm 0.08 \%$, respectivamente. Para el contenido de proteína los valores promedio oscilaron entre 5.40 y $6.28 \%$. El mayor valor se tuvo la primera semana de lactancia, para caer de forma rápida del día 21 al 35 posparto (5.40 a $5.45 \%$ ), momento a partir del cual se observó un incremento paulatino de esta variable hasta el día 91 del muestreo (5.71\%). El porcentaje de grasa en la leche mostró las mayores variaciones, se observa incrementó de forma paulatina con el transcurso de la lactancia, desde $1.66 \%$ al inicio hasta $3.79 \%$ al final en la semana 12. En contraste, el contenido de lac- 
Tabla 2. Promedio ( \pm error estándar) para los componentes químicos de la leche de ovejas Criollas Obispo registrados bajo condiciones semi-intensivas de trópico seco.

\begin{tabular}{lcccc}
\hline Característica & $\bar{X}+e e$ & Mínimo & Máximo & Coeficiente de variación \\
\hline Proteína (\%) & $5.73 \pm 0.07$ & 5.08 & 6.31 & 6.15 \\
Grasa (\%) & $2.49 \pm 0.19$ & 1.66 & 4.79 & 35.71 \\
Lactosa (\%) & $4.59 \pm 0.01$ & 4.48 & 4.70 & 1.29 \\
Sólidos totales (\%) & $13.66 \pm 0.19$ & 12.19 & 15.85 & 6.69 \\
Sólidos no grasos (\%) & $11.24 \pm 0.08$ & 10.42 & 11.82 & 3.60 \\
\hline
\end{tabular}

tosa presentó poca variación durante el periodo de muestreo, entre el registro mínimo (4.48\%) y máximo $(4.70 \%)$ con promedio de $4.59 \%$. En tanto que el contenido de sólidos totales tuvo un promedio de $13.66 \%$, con valor al principio de la lactancia de $13.37 \%$ y descenso en la semana dos (12.55\%) y tres $(12.46 \%)$, para posteriormente repuntar en la semana 12 después del parto (15.85\%). El porcentaje de sólidos no grasos promedio fue de $11.24 \%$, con comportamiento errático, pero dentro de un rango estrecho (10.42 a 11.82\%), a lo largo de la lactancia. Al inicio se tuvo un valor promedio de $11.76 \%$ que disminuyó en la semana tres $(10.90 \%)$, cuatro $(10.94 \%)$ y cinco (10.91\%), para posteriormente incrementar hasta alcanzar un valor de $11.27 \%$, en la última semana de muestreo.

De acuerdo con los valores encontrados por Delacroix-Buchet et al. (1994), Hadjipanayiotou (1995), Bencini y Pulina (1997), Park et al. (2007) y Reynal et al. (2008) para diferentes razas de ovinos cárnicas, de doble propósito o productoras de leche, los componentes de la leche para esta especie se encuentran dentro de los rangos para grasa de 3.60 a $9.97 \%$, lactosa de 4.11 a $5.51 \%$, proteína de 4.75 a $7.20 \%$, sólidos no grasos de 11.0 a $12 \%$ y sólidos totales de 14.4 a $20.7 \%$. Con excepción del porcentaje de grasa, los demás componentes de la leche se encuentran dentro de los rangos reportados en la literatura. Este bajo valor en el porcentaje de grasa puede atribuirse al hecho de se ha documentado que los ovinos Criollos almacenan mayor cantidad de grasa peri-renal en comparación con otras razas, lo cual parece ser una reserva energética para hacer uso de ella en momentos de restricción de alimento, y favorecer la sobrevivencia en épocas de estiaje severo (Solís et al. 2015). Esta misma condición de menor contenido de grasa en la leche también ha sido encontrada en la borrega Criolla Chiapas (4.37\%); pero los demás componentes químicos obtenidos en la leche de ovejas Obispo, son similares a los valores de proteína, sólidos no grasos totales y lactosa determinados en la oveja Chiapas (Carrillo-Pineda et al. 2015). Con excepción del menor contenido de grasa, las estimaciones de los demás componentes químicos de la leche de la oveja Obispo son cercanas a las informadas por Bencini y Purvis (1990) y Bencini y Pulina (1997) en las razas españolas consideradas como antecesoras de los ovinos Criollos de México (Solís et al. 2015). Para el contenido de proteína Bencini y Purvis (1990) en leche de ovejas de la raza Merina reportan valores entre 3.79 y $6.75 \%$ en las primeras y últimas semanas de lactación. En contraste, parece que la lactosa es el único componente que aumenta al principio de la lactación, para luego mantenerse constante y disminuir en el último tercio de la misma. El contenido de lactosa aumentó hasta la tercera semana de lactación, alcanzando un $5.87 \%$, para luego tener una tendencia decreciente. Al respecto, Hadjipanayiotou (1995) en la oveja Chios observó que la lactosa aumentó desde el día del parto hasta los 11 días posteriores para posteriormente mantenerse constante el resto de la lactancia. Un comportamiento similar para estos componentes fue observado en los registros semanales de composición química obtenidos durante los primeros tres meses posparto del presente estudio.

La curva de producción de leche en la oveja Obispo exhibe un pico entre la segunda y tercera semana después del parto, que es similar al observado en otras razas de ovinos. Su producción diaria promedio es cercana a la encontrada en la borrega Criolla Chiapas, pero inferior a la reportada para otras razas de ovinos. Con excepción del menor porcentaje del contenido de grasa, los valores promedio de los 
demás componentes se encuentran dentro del rango reportado en la literatura. Dado que el nivel de producción de leche que muestra la oveja en las primeras semanas posparto determina la sobrevivencia de la cría hasta el momento del destete, el interés principal en los genotipos destinados al consumo de carne, reside en el incremento de partes específicas del cordero como el músculo, hueso y grasa. En este sentido, es de importancia evaluar la forma en que la producción de leche de la oveja y su composición química influyen en el crecimiento del cordero du- rante la lactancia.

\section{AGRADECIMIENTOS}

Los resultados forman parte del Proyecto No. 176388 Convocatoria CB-2012-01, financiado por el Fondo Sectorial para la Educación SEP-CONACYT titulado "Caracterización Morfo-estructural, Reproductiva y Genética del Borrego de Cuatro Cuernos de la Montaña de Guerrero" y de la tesis de licenciatura del primer autor.

\section{LITERATURA CITADA}

Bencini R, Pulina G (1997) The quality of sheep milk: A review. Australian Journal of Experimental Agriculture 37: 485-504.

Bencini R, Purvis IW (1990) The yield and composition of milk from Merino sheep. Proccedings of the Australian Society of Animal Production 18: 144-147.

Bencini R, Hartmann PE, Lightfoot RJ (1992) Comparative dairy potential of Awassi x Merino ewes. Proccedings of the Australian Association of Animal Breeding and Genetics 10: 114-117.

Benchohra M, Amara K, Heminda H, Kalbaza AY, Aggad H (2013) Assessing dairy potential and lamb growth performance in Algerian Rembi sheep. Livestock Research Rural Development, Volume 25, Article 218.

Carrillo-Pineda KN, López-Ordaz R, Méndez-Gómez ADC, Peralta-Lailson M, Ulloa-Arvizu R, et al. (2015) Milk composition of Chiapas sheep breed under grazing conditions. International Journal of Livestock Production 6: $16-25$.

Castellanos RA, Valencia ZM (1982) Quantitative and qualitative study of milk production of the Pelibuey sheep. Tropical Animal Production 7: 323-240.

Chang Y, Rekaya R, Gianola D, Thomas DL (2001) Genetic variation of lactation curves in dairy sheep: a Bayesian analysis of Wood's function. Livestock Production Science 71: 241-251.

Chang Y, Rekaya R, Gianola D, Thomas DL (2002) Genetic variation of lactation curves in dairy sheep: quadratic function versus Wood's model. Livestock Production Science 77: 147-153.

Cornejo OZ, Rebolledo VR (2016) Estimación de parámetros en modelos no lineales: algoritmos y aplicaciones. Revista EIA 13: 81-98.

Delacroix-Buchet A, Barrillet F, Lagriffoul G (1994) Caractérisation de l'aptitude fromagère des lait de brebis Lacaune a l'aide d'un fromage. Lait 74: 173-186.

Delgadillo ADMC (2016) Lactancia: la firma de los mamíferos. Apuntes de Ciencia \& Sociedad 6: 62-69.

Doney JH, Peart JN, Smith WF, Londa F (1979) A consideration of the techniques for estimation of milk yield by sucking sheep and comparison of estimate obtained by two methods in relation to the effect of breed, level of production and stage of lactation. Journal of Agricultural Science Cambridge 92: 123-132.

Fasulkov IR (2012) Ultrasonography of the mammary gland in ruminants: a review. Bulgarian Journal of Veterinary Medicine 13: 1-12. 
García ME (1988) Modificaciones al sistema de clasificación climática de Köppen para adaptarlo a las condiciones de la República Mexicana. Instituto de Geografía, Universidad Nacional Autónoma de México. México. 217p.

Hadjipanayiotou M (1995) Composition of ewe, goat and cow milk and of colostrum of ewes and goats. Small Ruminant Research 18: 255-262.

Keskin I, Dağ B (2006) Comparison of different mathematical models for describing the complete lactational of Akkaraman ewes in Turkey. Asian-Australasian Journal of Animal Sciences 19: 1551-1555.

Labussière $\mathrm{J}$ (1988) Review of physiological and anatomical factors influencing the milking ability of ewes and the organization of milking. Livestock Production Science 18: 253-273.

Martínez RRD (2016) El borrego “Obispo” de la Montaña de Guerrero. Elementos 103: 35-39.

Martínez-Rojero RD, Mastache-Lagunas AA, Ulloa-Arvizu R, Reyna-Santamaría L, Zarco-Quintero LA, MejíaVillanueva O (2016) Caracterización morfológica en dos variedades de ovinos Criollos de la Montaña de Guerrero. Revista Mexicana de Agroecosistemas 3: 34-36.

Nudda A, Pulina G, Vallebella R, Bencini R, Enne G (2000) Ultrasound technique for measuring mammary cistern size of dairy ewes. Journal Dairy Research 67: 101-106.

Ochepo GO, Ayoade JA, Attah S, Adenkola AY (2015) Effect of breeds and method of milking on yield and composition of sheep milk. Scholarly Journal of Agricola Science 5: 232-235.

Ochoa CMA, Torres HG, Ochoa AAE, Vega RL, Mandeville PB (2002) Milk yield and composition of Rambouilliet ewes under intensive management. Small Ruminant Research 43: 269-274.

Park Y W, Juárez-Ramos M, Haenlein GFW (2007) Physicochemical characteristics of goat and Sheep milk. Small Ruminant Research 68: 88-113.

Pedraza P, Peralta M, Perezgrovas R (1992) El borrego Chiapas: una raza local mexicana de origen español. Archivos de Zootecnia 41: 355-362.

Peralta-Lailson M, Trejo-González AA, Pedraza-Villagómez P, Berruecos-Villalobos JM, Peláez VC (2005) Factors effecting milk yield and lactation curve fitting in the creole sheep of Chiapas-Mexico. Small Ruminant Research 58: 265-273.

Raynal LK, Lagriffoul G, Paccard P, Guillet I, Chiliard Y (2008) Composition of goat and sheep milk products: An updated. Small Ruminant Research 79: 57-72.

Reynolds LL, Brown L (1991) Assesing dairy potential of western White-Faced ewes. Journal of Animal Science 69: $1354-1362$.

SAS (2016) Base 9.4 Procedures guide. SAS Institute Inc, Cary NC. 556p.

Solís RJ, Romero EE, Rivera SMB (2015) Los recursos zootécnicos criollos en México: Un recurso necesario. Ovinos. $1^{\text {a }}$ Edición. BBA Biblioteca Básica de Agricultura. Colegio de Postgraduados. México. 188p.

Steel RGD, Torrie JH (1989) Bioestadística: Principios y procedimientos. Segunda edición en español. Editorial McGraw-Hill, México. 622p.

Wood PDP (1976) Algebraic models of the lactation curves for milk, fat and protein production, with estimates of seasonal variation. Animal Production 22: 35-40. 